\title{
LINEAR STABILITY OF HOMOGENEOUS RICCI SOLITONS
}

\author{
CHRISTINE GUENTHER, JAMES ISENBERG, AND DAN KNOPF
}

\begin{abstract}
As a step toward understanding the analytic behavior of Type-III Ricci flow singularities, i.e. immortal solutions that exhibit $|\mathrm{Rm}| \leq C / t$ curvature decay, we examine the linearization of an equivalent flow at fixed points discovered recently by Baird-Danielo and Lott: nongradient homogeneous expanding Ricci solitons on nilpotent or solvable Lie groups. For all explicitly known nonproduct examples, we demonstrate linear stability of the flow at these fixed points and prove that the linearizations generate $C_{0}$ semigroups.
\end{abstract}

\section{INTRODUCTION}

In [19, the authors investigate the stability of compact flat and Ricci-flat solutions of Ricci flow - where there is a center manifold present in the space of Riemannian metrics - by a method incorporating the linearized Ricci flow operator, the analytic semigroup it generates, and appropriate interpolation spaces. (One of our results has recently been strengthened by Šešum using somewhat different techniques; see [45].)

The work described in this note concerns a related question: stability along a collapsing locally homogeneous solution of Ricci flow. One incentive for considering this more nuanced issue arises when studying the geometrization of a closed 3 -manifold. The idea is essentially as follows. (See Section 2 for a more precise discussion.) Let $\mathcal{M}_{t}^{3}$ denote the (possibly disconnected) solution of Ricci flow-withsurgery at some non-surgery time $t \gg 0$. A central claim of the Hamilton-Perelman Geometrization program is that there exists a decomposition $\mathcal{M}_{t}^{3}=\mathcal{M}_{\text {thick }}^{3} \cup \mathcal{M}_{\text {thin }}^{3}$. (See [41, 42], 30], and [11.) $\mathcal{M}_{\text {thick }}^{3}$ consists of those components where the injectivity radius is controlled in a suitable manner; these components should be close in a precise sense to a finite collection of complete finite-volume hyperbolic 3 -manifolds, truncated along cuspidal tori whose images are incompressible in $\mathcal{M}_{t}^{3}$. (Compare 24].) The components of $\mathcal{M}_{\text {thin }}^{3}$ are collapsed with local lower bounds on sectional curvature; these components should be Cheeger-Gromov graph manifolds. (See [46, 47].) A graph manifold in the sense of Cheeger-Gromov [7, 8] is a closed 3-manifold admitting a decomposition by not necessarily incompressible tori such that each complementary piece is a Seifert space. Any such manifold either is a topological graph manifold (i.e. it may be decomposed along incompressible tori such that each complementary piece is a Seifert manifold) or else is a connected sum of topological graph manifolds with lens space and $\mathcal{S}^{1} \times \mathcal{S}^{2}$ factors.

Many intriguing questions remain open about the behavior of Ricci flow on $\mathcal{M}_{\text {thin }}^{3}$. A long-standing question of Hamilton asks whether the Ricci flow on these components may be modeled in a suitable manner by its behavior on the homogeneous Thurston model geometries. If so, one would expect that the Ricci flow of

Revised September 5, 2006. 
the locally homogeneous Thurston geometries - among which nil and sol exhibit Cheeger-Gromov collapse - should in an appropriate sense be stable in the space of 3-dimensional solutions. (See [27] and [32], as well as [25] and [31].)

Recent work of Lott [37. allows a refinement of Hamilton's question. In any dimension, a Type-III solution of Ricci flow $\left(\mathcal{M}^{n}, g(t)\right)$ is one that exists for $t \in$ $[0, \infty)$ and satisfies $\sup _{\mathcal{M}^{n} \times[0, \infty)} t|\operatorname{Rm}|<\infty$. Given such a solution, fix an origin $x \in \mathcal{M}^{n}$. Then for each $s>0$, there is a rescaled pointed solution of Ricci flow $\left(\mathcal{M}^{n}, g_{s}(t), x\right)$ defined for all $t \in[0, \infty)$ by $g_{s}(t):=s^{-1} g(s t)$. If the original solution is a finite-volume locally homogeneous 3 -manifold, Lott proves that $\lim _{s \rightarrow \infty} g_{s}(\cdot)$ exists as a homogeneous Ricci soliton on a 3 -dimensional étale groupoid 1 (Compare [18.) Let $\tilde{g}(\cdot)$ denote the lift of $g(\cdot)$ to the universal cover $\tilde{\mathcal{M}}^{3}$. The limit solution on $\tilde{\mathcal{M}}_{\infty}^{3}$ is $\tilde{g}_{\infty}(t):=\lim _{s \rightarrow \infty}\left(\psi_{s}^{*}\left(s^{-1} \tilde{g}(s t)\right)\right)$, where $\left\{\psi_{s}\right\}$ is a family of time-independent diffeomorphisms. Thus $\tilde{g}_{\infty}(\cdot)$ encodes the asymptotic geometry of $\tilde{g}(\cdot)$ at large times and large length scales 3 Lauret 34 has shown that, in many cases, a homogeneous soliton is essentially unique 3 Any such soliton is a fixed point of the transformation $\tilde{g}(\cdot) \mapsto \tilde{g}_{\infty}(\cdot)$. Therefore, one implication of Lott's results may be interpreted as the statement that if a fixed 3-dimensional Lie group admits a homogeneous Ricci soliton - which is true for nil and sol, but not for $\widetilde{\mathrm{SL}}(2, \mathbb{R})$ - then the soliton acts in this sense as an infinite-time attractor among all solutions respecting the group symmetry. From the point of view of Hamilton's original question, one may further ask whether a given homogeneous Ricci soliton acts as an infinite-time attractor among all nearby solutions, symmetric or not. If so, then the collapsing behavior of the soliton is stable for Ricci flow, in a precise sense.

For example, suppose $\left(\mathcal{M}^{3}, g(t)\right)$ is a locally homogeneous nil-geometry solution of Ricci flow on a compact manifold. Lifting to the universal cover $\tilde{\mathcal{M}}^{3} \approx \mathbb{R}^{3}$, one finds homogeneous nil-geometry metrics $\left(\tilde{\mathcal{M}}^{3}, G(t)\right)$ evolving by Ricci flow. In the six-dimensional family $4 \mathcal{G}$ parameterizing such evolutions, exactly one is a soliton 5 Call this $G_{0}(\cdot)$, the lift of $g_{0}(\cdot)$. (Note that $G_{0}(\cdot)$ is unique up to scaling, and that $g_{0}(\cdot)$ is a locally homogeneous nil-geometry solution.) Lott shows that the solution determined by the initial datum $G_{0}(0)$ is stable within $\mathcal{G}$, in a precise sense. Is there anything one can say about the stability of $G_{0}(0)$ among its non-homogeneous perturbations $\tilde{G}$, or of $g_{0}(0)$ among its non-locally-homogeneous perturbations $\tilde{g}$ ?

In this note, we explore an approach to these questions from the point of view of [19]. A homogeneous Ricci soliton may be regarded as a fixed point of a related flow, solutions of which are in one-to-one correspondence with solutions of Ricci flow. If one could show that the linearization of this flow obeys a spectral bound

\footnotetext{
${ }^{1}$ More generally, Lott's compactness theorem [37. Theorem 1.4] shows that any sequence $\left(\mathcal{M}_{k}^{n}, g_{k}(\cdot), x_{k}\right)$ of complete pointed Ricci flows defined on a common time interval and obeying uniform curvature bounds subconverges to a solution of Ricci flow on a pointed $n$-dimensional étale groupoid. In general, one does not know this is a soliton.

${ }^{2}$ The limit $\tilde{g}_{\infty}(\cdot)$ should be compared to the asymptotic gradient shrinking soliton of an ancient $\kappa$-solution, which Perelman constructs [41, §11.2] as a blow-down limit as $t \rightarrow-\infty$.

${ }^{3}$ See Theorem 4 below.

${ }^{4} \mathrm{~A}$ homogeneous solution of Ricci flow is determined by its initial datum, a left-invariant metric. A left-invariant metric on an $n$-dimensional homogeneous space is determined by its value on any tangent space, hence may be identified with a positive-definite symmetric $(n \times n)$-matrix. Any such matrix is determined by $n(n+1) / 2$ parameters.

${ }^{5}$ Uniqueness was proved by Lauret [34]. Explicit constructions were done independently by Baird-Danielo [3] and Lott 37. See Sections 2 and 3 for a more thorough exposition.
} 
and generates an analytic semigroup, then results of Da Prato and Lunardi 12 , Theorem 2.2] would imply asymptotic stability of the soliton among all nearby solutions of Ricci flow in a suitable interpolation space. However, for the solitons under consideration, the analytic and technical challenges of this program are formidable. The linearizations of the modified flow at these solitons are second-order tensor-valued differential operators with quadratically unbounded first and zeroth order coefficients posed on noncompact manifolds. These operators are not self adjoint in any weighted $L^{2}$ space. Such operators have substantial independent interest from the perspective of analysis. Indeed, the analysis of scalar-valued operators with unbounded coefficients is a very active area of current research, because of their connection with Ornstein-Uhlenbeck operators with unbounded drift and Schrödinger operators with unbounded potentials. For a small sampling, see 9 , [10, 20], 36], 39], and [44].

From the point of view of analysis, the key results of this paper concern certain geometrically motivated second-order non-self-adjoint tensor-valued differential operators with quadratically unbounded lower-order coefficients on noncompact manifolds. We show that these operators generate strongly continuous semigroups and that their spectra have strictly negative (i.e. stable) real parts.

This note is organized as follows. In Section 2 we provide a more thorough background and motivation for studying the asymptotic stability of expanding homogeneous solitons. In Section 3, we derive the modified Ricci flow (3.2) and its linearization (3.4) at soliton fixed points, with an eye toward studying this stability. In Section 4, we discuss all three explicitly known nonproduct examples: two are nilsolitons and one exhibits sol geometry. We prove linear stability of the modified Ricci flow at these fixed points. (See Propositions 4, 6, and 8.) This provides a formal argument in support of asymptotic stability. In Section 5 , we prove that the linear operators generate $C_{0}$ semigroups. (See Theorem 5) Even this result is far from trivial and may be of independent analytic interest, as we have explained in the previous two paragraphs. Our progress in this note falls short of establishing analytic semigroups. So the question of asymptotic stability remains open but appears promising, in light of our results in Sections 4 and 5 . We hope that our conclusions here facilitate further study of this interesting area of geometric analysis. In Section 6, we briefly discuss some directions for further research.

Acknowledgments. C.G. is partially supported by the Thomas J. and Joyce Holce Professorship in Science. J.I. is partially supported by NSF grant PHY-0354659. D.K. is partially supported by NSF grants DMS-0511184 and DMS-0505920.

D.K. thanks Sigurd Angenent and Rafe Mazzeo for their interest and discussions. The authors thank the referee for suggestions to improve the exposition.

\section{Motivation AND BACKGROUnd}

2.1. Ricci soliton structures. A Ricci soliton structure consists of the data $\left(\mathcal{M}^{n}, g, X, \alpha\right)$, where $\mathcal{M}^{n}$ is a smooth connected manifold, $g$ is a complete Riemannian metric on $\mathcal{M}^{n}, X$ is a complete vector field on $\mathcal{M}^{n}$, and $\alpha$ is a constant such that

$$
2 \mathrm{Rc}+\alpha g+\mathcal{L}_{X} g=0 .
$$

One says the soliton is shrinking, steady, or expanding if $\alpha<0, \alpha=0$, or $\alpha>0$, respectively. If $\eta_{t}$ is the one-parameter family of diffeomorphisms generated by the 
vector fields $Y(\cdot, t)=(1+\alpha t)^{-1} X(\cdot)$, then $\bar{g}(\cdot, t):=(1+\alpha t) \eta_{t}^{*} g(\cdot)$ is a solution of Ricci flow that evolves self similarly, i.e. only by scaling and diffeomorphism. Conversely, after normalization, every self similar solution of Ricci flow arises in this manner.

2.2. Solitons as finite-time singularity models. A major reason for studying shrinking or steady solitons is the information they can provide about finite-time singularities. If $\left(\mathcal{M}^{n}, g(t)\right)$ is a compact solution of Ricci flow that exists up to a maximal time $T<\infty$, one may study the developing singularity by forming a sequence of pointed dilated solutions $\left(\mathcal{M}^{n}, g_{k}(t), x_{k}\right)$, where $g_{k}(t)=\lambda_{k} g\left(t_{k}+t / \lambda_{k}\right)$ for some sequence of positive constants $\lambda_{k} \rightarrow \infty$. To get a complete, smooth limit, one takes $t_{k} \nearrow T$ and chooses the dilation factors $\lambda_{k}$ to be comparable to the supremum of $|\mathrm{Rm}|$ over appropriate space-time neighborhoods of $\left(x_{k}, t_{k}\right)$. Under quite general conditions, the injectivity radius estimate given by Perelman's No Local Collapsing Theorem [41] then allows application of Hamilton's Compactness Theorem [23] to show that the sequence $\left(\mathcal{M}^{n}, g_{k}(t), x_{k}\right)$ subconverges, modulo time-independent diffeomorphisms $\psi_{k}$, to a pointed limit solution of Ricci flow $\left(\mathcal{M}_{\infty}^{n}, g_{\infty}(t), x_{\infty}\right)$, called a singularity model. In dimension three, every finite-time singularity admits gradient solitons among its possible singularity models. (See 22 and 41 .) In any dimension, it is reasonable to expect finite-time singularity models to be shrinking or steady solitons. For example, the neckpinch singularity [1, 2] is modeled by the shrinking gradient soliton $\left(\mathbb{R} \times S^{n}, d s^{2}+2(n-1) g_{\text {can }}, \operatorname{grad}\left(s^{2} / 4\right),-1\right)$ for all $n \geq 2$.

2.3. Solitons as infinite-time singularity models. Our interest in this note is in immortal solutions of Ricci flow. As noted above, a Type-III solution of Ricci flow $\left(\mathcal{M}^{n}, g(t)\right)$ is an immortal solution that satisfies $\sup _{\mathcal{M}^{n} \times[0, \infty)} t|\operatorname{Rm}|<\infty$. There are many interesting open questions about the analytic properties of TypeIII solutions in all dimensions $n \geq 3$.

For Type-III solutions, no uniform injectivity radius estimate is known. In fact, none is possible. For example, compact locally homogeneous manifolds with nil ${ }^{3}$ geometry occur as mapping tori of $\Upsilon_{k}: T^{2} \rightarrow T^{2}$ induced by $\left(\begin{array}{ll}1 & k \\ 0 & 1\end{array}\right) \in \operatorname{SL}(2, \mathbb{Z})$ with $k \neq 0$. As shown in 27 and 32 , Ricci flow solutions with this geometry are all Type-III, with $|\operatorname{Rm}(g(t))| \leq C /(1+t)$ and with injectivity radius decaying like $t^{-1 / 6}$ as $t \rightarrow \infty$. Other three-dimensional examples are analyzed in [27] and [32, while four-dimensional examples are studied in [28].

Many of these homogeneous solutions exhibit pointed Gromov-Hausdorff convergence to lower dimensional manifolds. For such solutions, it is not possible to form a limit solution $\left(\mathcal{M}_{\infty}^{n}, g_{\infty}(t), x_{\infty}\right)$ in a naive way. Nonetheless, an old conjecture of Hamilton makes the following prediction.

Conjecture 1 (Hamilton). A Type-III solution should, in a suitable sense, behave asymptotically like a (locally) homogeneous model.

Evidence in favor of Hamilton's conjecture is found in prior work of the authors, specifically [25] and [31]. As noted above, further evidence comes from recent work of Lott [37. Let $\left(\mathcal{M}^{n}, g(t)\right)$ be a solution of Ricci flow, and let $\left(\mathcal{M}^{n}, g_{s}(t), x\right)$ denote the rescaled pointed solution of Ricci flow defined for $t \in[0, \infty)$ by $g_{s}(t)=\frac{1}{s} g(s t)$.

Theorem 1 (Lott). Let $\left(\mathcal{M}^{n}, g(t)\right)$ be a Type-III solution of Ricci flow. If the limit

$$
\left(\mathcal{M}_{\infty}^{n}, g_{\infty}(t), x_{\infty}\right)=\lim _{s \rightarrow \infty}\left(\mathcal{M}^{n}, g_{s}(t), x\right)
$$


exists, then $\left(\mathcal{M}_{\infty}^{n}, g_{\infty}(t)\right)$ is an expanding Ricci soliton.

Bear in mind that this convergence is modulo (time-independent) diffeomorphisms $\psi_{s}$, which one should not expect to converge as $s \rightarrow \infty$. Note too that the word 'expanding' refers to the sign of $\alpha$ in (2.1), hence is compatible with collapse.

In dimension $n=3$, one can make a stronger statement, one that does not need convergence as a hypothesis.

Theorem 2 (Lott). Let $\left(\mathcal{M}^{3}, g(t)\right)$ be a solution of Ricci flow on a simply-connected homogeneous space $\mathcal{M}^{3}=G / K$, where $G$ is a unimodular Lie group and $K$ is a compact isotropy subgroup. Then there exists a limit

$$
\left(\mathcal{M}_{\infty}^{3}, g_{\infty}(t), x_{\infty}\right)=\lim _{s \rightarrow \infty}\left(\mathcal{M}^{3}, g_{s}(t), x\right)
$$

which is an expanding homogeneous soliton (on a possibly different Lie group).

In light of Theorems 1 and 2, it is natural to refine Conjecture 1 by comparing the behavior of an arbitrary Type-III solution to an expanding homogeneous soliton.

2.4. Expanding homogeneous solitons. Cheeger-Fukaya-Gromov show that any Riemannian manifold $\left(\mathcal{M}^{n}, g\right)$ which collapses with bounded curvature is close to one with a nilpotent structure. More precisely, there is for any $\varepsilon>0$ a metric $g_{\varepsilon}$ on $\mathcal{M}^{n}$ which is $C^{1}$-close to $g$ such that $g_{\varepsilon}$ admits a sheaf of nilpotent Lie algebras of local Killing vector fields acting in the collapsed directions [6]. (Also see [7, 8] and [16, 17.) For this reason, when looking for homogeneous models of Type-III behavior, it is natural to begin by seeking expanding Ricci soliton structures on nilpotent Lie groups, which we call Ricci nilsolitons.

This search has an interesting history. Lauret 34 approaches it by asking a different question: is there a 'best' $G$-invariant Riemannian metric on a given homogeneous space $G$ ? An Einstein metric would be an obvious candidate. Such metrics exist on some solvable Lie groups. In fact, every known example of a noncompact, nonflat homogeneous space that admits an Einstein metric is isomorphic to a solvable Lie group $S$ with a metric of 'standard type'. See [26] and [4]. (A left-invariant metric $g$ on a solvable Lie group $S$, regarded as an inner product on the Lie algebra $\mathfrak{s}$, is said to be of standard type if the orthogonal complement with respect to $g$ of the derived algebra $[\mathfrak{s}, \mathfrak{s}]$ forms an abelian subalgebra $\mathfrak{a}$ of $\mathfrak{s}$.)

On the other hand, a noncommutative, nilpotent Lie group admits no Einstein metric whatsoever. Indeed, by a result of Milnor, if the Lie algebra $\mathfrak{g}$ of $G$ is nilpotent but noncommutative, then the Ricci curvature of any left-invariant metric on $G$ has mixed sign [38, Theorem 2.4]. Since (2.1) reduces to $2 \mathrm{Rc}=-\alpha g$ when $X=0$, a Ricci soliton may be regarded as a generalization of an Einstein metric, and thus provides a substitute candidate for the role of 'best' metric. Lauret proves the following result.

Theorem 3 (Lauret). Let $N^{n}$ be a simply-connected nilpotent Lie group with Lie algebra $\mathfrak{n}$ and a left-invariant metric $g$. Then $\left(N^{n}, g\right)$ admits a Ricci soliton structure if and only if $(\mathfrak{n}, g)$ admits a standard metric solvable extension $(\mathfrak{s}=\mathfrak{a} \oplus \mathfrak{n}, \tilde{g})$ such that the simply-connected solvable Lie group $(S, \tilde{g})$ is Einstein.

If $\mathfrak{n}$ is a Lie algebra with inner product $g$, a standard metric solvable extension of $(\mathfrak{n}, g)$ is $(\mathfrak{s}, \tilde{g})$, where $\mathfrak{s}=\mathfrak{a} \oplus \mathfrak{n}$ is a solvable Lie algebra such that $\left.[\cdot, \cdot]_{\mathfrak{s}}\right|_{\mathfrak{n} \times \mathfrak{n}}=[\cdot, \cdot]_{\mathfrak{n}}$, and $\tilde{g}$ is an inner product of standard type such that $\left.\tilde{g}\right|_{\mathfrak{n} \times \mathfrak{n}}=g$ and $[\mathfrak{s}, \mathfrak{s}]_{\mathfrak{s}}=\mathfrak{n}=\mathfrak{a}^{\perp}$. 
As we noted above, the space of left-invariant metrics on $N^{n}$ has dimension $n(n+1) / 2$. However, a Ricci soliton structure, if one exists, is essentially unique.

Theorem 4 (Lauret). Let $N^{n}$ be a simply-connected nilpotent Lie group with Lie algebra $\mathfrak{n}$. If $g$ and $g^{\prime}$ are Ricci soliton metrics, then there exist $a>0$ and $\eta \in$ Aut $(\mathfrak{n})$ such that $g^{\prime}=a \eta(g)$.

Although its proof is nonconstructive, Theorem 3 provides many examples of existence or nonexistence. Any generalized Heisenberg group [4] and many other two-step nilpotent Lie groups admit a Ricci soliton structure. On the other hand, if $\mathfrak{n}$ is characteristically nilpotent, then $N^{n}$ admits no such structure. (See [34] and [35] for more examples.)

The first explicit constructions of Ricci soliton structures on nilpotent (or more generally, solvable) Lie groups are obtained by Baird and Danielo 3 in dimension $n=3$ and independently by Lott [37] in dimensions $n=3,4$. Remarkably, these are the first known examples of nongradient soliton structures. See equations (4.1), (4.4), and (4.7), below.

If $(N, g, X, \alpha)$ is an expanding Ricci soliton structure on a Lie group that admits a compact quotient $\mathcal{M}^{n}=N / K$, what can one say about soliton structures on $\mathcal{M}^{n}$ ? Because the metric $g$ is homogeneous, it is compatible with any compact quotient. But the soliton structure will never be compatible with compactification, because any compact steady or expanding soliton is Einstein. (See 29] or [22.) Accordingly, we call such compact quotients pseudosolitons - compact manifolds with no soliton structure which acquire one when lifted to an infinite cover. Apart from the BairdDanielo and Lott examples, the only other explicit example we know is $S^{1} \times S^{n}$ with a product metric. This is not a soliton for any $n \geq 2$, but it is a quotient of $\left(\mathbb{R} \times S^{n}, d s^{2}+2(n-1) g_{\text {can }}, \operatorname{grad}\left(s^{2} / 4\right),-1\right)$. Pseudosolitons open the door to a more complete understanding of the analytic behavior of Type-III solutions, and in particular of Conjecture 1. That is to say, if Hamilton's expectation is true, then nilpotent (or more generally, solvable) pseudosolitons should act in some sense as stable models of Type-III behavior.

Conjecture 2. Let $\left(\mathcal{M}^{n}, g(t)\right)$ be a solution of Ricci flow such that $g(0)$ is sufficiently close (in a suitable norm) to a (locally) homogeneous metric on (a quotient of) a nilpotent Lie group. Then there exists a limit solution $\left(\mathcal{M}_{\infty}^{n}, g_{\infty}(t), x_{\infty}\right)$ which is a nilsoliton 6

If $\left(\mathcal{M}^{n}, g(t)\right)$ is a solution on a compact manifold, properties of $\left(\mathcal{M}_{\infty}^{n}, g_{\infty}(t), x_{\infty}\right)$ should provide information about $\left(\mathcal{M}^{n}, g(t)\right)$ via equivariant convergence theory, for example, Lott's Riemannian groupoid results [37. and potential extensions.

\section{A MOdified RICCi FLOW AND ITS LiNEARIZATION}

3.1. The flow. Suppose one is given an immortal solution $\left(\mathcal{M}^{n}, \hat{g}(t)\right)$ of Ricci flow

$$
\frac{\partial}{\partial t} \hat{g}=-2 \operatorname{Rc}(\hat{g}), \quad(\beta<t<\infty)
$$

a constant $\alpha>0$, and a time-independent vector field $X$ on $\mathcal{M}^{n}$. Define a scaling factor

$$
\sigma(t)=\alpha(t-\beta)
$$

\footnotetext{
${ }^{6}$ One expects the nilsoliton to be a solution of Ricci flow on $\mathcal{M}_{\infty}^{n}=\tilde{\mathcal{M}}^{n}$ obtained as a limit of large-time asymptotic blow-downs, modulo diffeomorphisms, as in 37.
} 
and a one-parameter family of vector fields

$$
Y(x, t)=\frac{1}{\sigma(t)} X(x) .
$$

Let $\eta_{t}$ denote the family of diffeomorphisms of $\mathcal{M}^{n}$ generated by $Y$, so that

$$
\frac{\partial}{\partial t} \eta_{t}(x)=Y\left(\eta_{t}(x), t\right)=\frac{1}{\sigma(t)} X\left(\eta_{t}(x)\right) \text {. }
$$

Assume that $\eta_{t}$ exists for $t>\beta$. Because we are not assuming that $\mathcal{M}^{n}$ is compact, this assumption requires verification in our applications. (See Propositions [3] 5] and 7 below.) Let

$$
\tau(t)=\frac{1}{\alpha} \log (t-\beta),
$$

noting that $d \tau / d t=1 / \sigma(t)$. Define a one-parameter family of metrics $g(\tau)$ on $\mathcal{M}^{n}$ by

Observe that

$$
g(\tau)=\frac{1}{\sigma\left(\beta+e^{\alpha \tau}\right)}\left(\eta_{\beta+e^{\alpha \tau}}^{-1}\right)^{*}\left[\hat{g}\left(\beta+e^{\alpha \tau}\right)\right]
$$

and that

$$
\hat{g}(t)=\sigma(t) \eta_{t}^{*}[g(\tau(t))]
$$

$$
\begin{aligned}
\frac{\partial}{\partial t} \hat{g}(t) & =\sigma^{\prime}(t) \eta_{t}^{*}[g(\tau(t))]+\sigma(t) \eta_{t}^{*}\left\{\mathcal{L}_{Y(t)} g(\tau(t))+\frac{d \tau}{d t}\left[\frac{\partial}{\partial \tau} g(\tau)\right]\right\} \\
& =\eta_{t}^{*}\left\{\alpha g(\tau)+\mathcal{L}_{X} g(\tau)+\frac{\partial}{\partial \tau} g(\tau)\right\} .
\end{aligned}
$$

Recalling that $\hat{g}(t)$ is a solution of Ricci flow, one sees that

$$
\eta_{t}^{*}\{-2 \operatorname{Rc}(g)\}=-2 \operatorname{Rc}(\hat{g})=\eta_{t}^{*}\left\{\alpha g(\tau)+\mathcal{L}_{X} g(\tau)+\frac{\partial}{\partial \tau} g(\tau)\right\},
$$

hence that $g(\tau)$ evolves by the modified Ricci flow

$$
\frac{\partial}{\partial \tau} g=-2 \operatorname{Rc}(g)-\mathcal{L}_{X} g-\alpha g, \quad(-\infty<\tau<\infty) .
$$

The following observation then follows immediately from (2.1).

Proposition 1. If $\left(\mathcal{M}^{n}, g, X, \alpha\right)$ is an expanding Ricci soliton structure, then $g$ is a fixed point of the modified Ricci flow (3.2).

Remark 1. This construction is easily adapted to shrinking $(\alpha<0)$ or steady $(\alpha=0)$ solitons.

3.2. The linearization. The linearization of (3.2) is entirely standard. We recall it here for the convenience of the reader.

Let $h$ be an arbitrary smooth (2,0)-tensor field with compact support on $\left(\mathcal{M}^{n}, g\right)$. Let $\{\tilde{g}(s):-\varepsilon<s<\varepsilon\}$ be a one-parameter family of metrics with $\tilde{g}(0)=g$ and

$$
\left.\frac{\partial}{\partial s} \tilde{g}\right|_{s=0}=h
$$

Define $H=\operatorname{tr}_{g} h$. It is well known that the linearization of $-2 \operatorname{Rc}(g)$ at $g$ is

$$
\left.\frac{\partial}{\partial s}\left(-2 R_{i j}\right)\right|_{s=0}=\Delta_{\ell} h_{i j}+\nabla_{i} \nabla_{j} H+\nabla_{i}(\delta h)_{j}+\nabla_{j}(\delta h)_{i},
$$


where $\Delta_{\ell}$ denotes the Lichnerowicz Laplacian

$$
\Delta_{\ell} h_{i j}=\Delta h_{i j}+2 R_{i p q j} h^{p q}-R_{i}^{k} h_{k j}-R_{j}^{k} h_{i k} .
$$

We impose ellipticity by the DeTurck trick 13, 14. Let $\{\tilde{X}(s):-\varepsilon<s<\varepsilon\}$ be a one-parameter family of smooth compactly supported vector fields with $\tilde{X}(0)=X$ and 7

Observing that 8

$$
\left.\frac{\partial}{\partial s} \tilde{X}\right|_{s=0}=W:=\left(\frac{1}{2} d H+\delta h\right)^{\sharp} .
$$

$$
\left.\frac{\partial}{\partial s}\left(\mathcal{L}_{\tilde{X}} \tilde{g}\right)\right|_{s=0}=\mathcal{L}_{W} g+\mathcal{L}_{X} h
$$

where $\left(\mathcal{L}_{W} g\right)_{i j}=\nabla_{i} \nabla_{j} H+\nabla_{i}(\delta h)_{j}+\nabla_{j}(\delta h)_{i}$, one reaches the following conclusion.

Proposition 2. The linearization of the modified Ricci flow (3.2) at a Ricci soliton $\left(\mathcal{M}^{n}, g, X, \alpha\right)$ is given by

where $L$ is the elliptic operator

$$
\frac{\partial}{\partial \tau} h=L h,
$$

$$
L: h \mapsto \Delta_{\ell} h-\mathcal{L}_{X} h-\alpha h .
$$

A priori, $L$ is defined only on smooth compactly supported tensor fields. Below, we will specify a domain that is useful for our purposes.

Remark 2. It is convenient to write $L$ in the alternative form

$$
L: h \mapsto \Delta_{\ell} h-\nabla_{X} h-\alpha h-\Xi(h),
$$

where in coordinates, $\Xi(h)_{i j}=\nabla_{i} X^{k} h_{k j}+\nabla_{j} X^{k} h_{k i}$.

Example 1. The Gaussian soliton is $\left(\mathbb{R}^{n}, g, X, \alpha\right)$, where $g$ is the standard flat metric, $\alpha \in \mathbb{R}$ is arbitrary, and

$$
X(x)=\operatorname{grad}\left(-\frac{\alpha}{4}|x|^{2}\right) .
$$

The linearization (3.4) is simply

$$
L: h \mapsto \Delta h-\nabla_{X} h,
$$

which is self adjoint in the weighted space $L^{2}\left(\mathbb{R}^{n} ; e^{\frac{\alpha}{4}|x|^{2}} d \mu\right)$, where $d \mu=d \mu(g)$.

Critically, it turns out that the linearization is self adjoint in a weighted space if and only if there is a gradient soliton structure, i.e. if and only if $X=\operatorname{grad} \varphi$ for some potential function $\varphi: \mathcal{M}^{n} \rightarrow \mathbb{R}$.

Remark 3. Let $L$ be the operator (3.4), let $\varphi$ be a smooth function, and let $(\cdot, \cdot)_{\varphi}$ denote the weighted $L^{2}$ inner product $(u, v)_{\varphi}=\int_{\mathcal{M}^{n}}\langle u, v\rangle e^{-\varphi} d \mu$. Then one has

$$
\begin{aligned}
(L u, v)_{\varphi}-(u, L v)_{\varphi} & =2\left(u, \nabla_{(X-\nabla \varphi)}\right)_{\varphi}+2\left(d X_{b}, u v\right)_{\varphi} \\
& -\int_{\mathcal{M}^{n}}\langle u, v\rangle\left\{\Delta \varphi+\nabla_{X} \varphi-|\nabla \varphi|^{2}+\delta X\right\} e^{-\varphi} d \mu,
\end{aligned}
$$

where $\left(d X_{b}\right)_{i j}=\nabla_{i} X_{j}-\nabla_{j} X_{i}$ in coordinates. Clearly, the right-hand side above vanishes if $X=\operatorname{grad} \varphi$. The converse is left as an exercise.

\footnotetext{
${ }^{7}$ If $\theta$ is a 1 -form, we denote by $\theta^{\sharp}$ the vector field metrically dual to $\theta$.

${ }^{8}$ This choice of $W$ is essentially equivalent to the Bianchi gauge, as adopted in the elliptic context by Biquard [5] and others.
} 


\section{SPECTRAL BOUNDS FOR KNOWN EXAMPLES}

4.1. nil $^{3}$ geometry. We now describe the nil ${ }^{3}$ soliton $\left(\mathcal{M}^{3}, g, X, 3\right)$ constructed in [3. (Also see [37, Section 3.3.3].) Because the exponential map of any connected, simply-connected, nilpotent Lie group is a diffeomorphism, $\mathcal{M}^{3}$ is diffeomorphic to $\mathbb{R}^{3}$. In standard coordinates $\left(x_{1}, x_{2}, x_{3}\right)$ on $\mathbb{R}^{3}$, consider the frame field $F=$ $\left(F_{1}, F_{2}, F_{3}\right)$ given by

$$
F_{1}=2 \frac{\partial}{\partial x_{1}}, F_{2}=2\left(\frac{\partial}{\partial x_{2}}-x_{1} \frac{\partial}{\partial x_{3}}\right), F_{3}=2 \frac{\partial}{\partial x_{3}} .
$$

It is easy to check that all brackets $\left[F_{i}, F_{j}\right]$ vanish except $\left[F_{1}, F_{2}\right]=-2 F_{3}$. The connection is represented by the matrix

$$
\left(\nabla_{F_{i}} F_{j}\right)=\left(\begin{array}{ccc}
0 & -F_{3} & F_{2} \\
F_{3} & 0 & -F_{1} \\
F_{2} & -F_{1} & 0
\end{array}\right) .
$$

With respect to the dual field

$$
\varphi^{1}=\frac{1}{2} d x_{1}, \varphi^{2}=\frac{1}{2} d x_{2}, \varphi^{3}=\frac{1}{2}\left(x_{1} d x_{2}+d x_{3}\right),
$$

one may identify $g=g_{i j} \varphi^{i} \otimes \varphi^{j}$ with the matrix

$$
\left(g_{i j}\right)=\left(\begin{array}{ccc}
4 & 0 & 0 \\
0 & 4 & 0 \\
0 & 0 & 4
\end{array}\right)
$$

Recalling the standard formula for the Riemannian curvature,

$$
\begin{aligned}
\langle R(X, Y) Y, X\rangle & =\frac{1}{4}\left|(\operatorname{ad} X)^{*} Y+(\operatorname{ad} Y)^{*} X\right|^{2}-\left\langle(\operatorname{ad} X)^{*} X,(\operatorname{ad} Y)^{*} Y\right\rangle \\
& -\frac{3}{4}|[X, Y]|^{2}-\frac{1}{2}\langle[[X, Y], Y], X\rangle-\frac{1}{2}\langle[[Y, X], X], Y\rangle,
\end{aligned}
$$

we compute the Ricci curvature tensor $\mathrm{Rc}=R_{i j} \varphi^{i} \otimes \varphi^{j}$ of $g$ and identify it with

$$
\left(R_{i j}\right)=\left(\begin{array}{ccc}
-2 & 0 & 0 \\
0 & -2 & 0 \\
0 & 0 & 2
\end{array}\right) \text {. }
$$

Define the vector field

$$
X=-\frac{1}{2} x_{1} F_{1}-\frac{1}{2} x_{2} F_{2}-\left(\frac{1}{2} x_{1} x_{2}+x_{3}\right) F_{3} .
$$

A calculation shows that $\nabla X=\nabla_{i} X^{j} \varphi^{i} \otimes F_{j}$ has components

$$
\left(\nabla_{i} X^{j}\right)=\left(\begin{array}{ccc}
-1 & -\left(\frac{1}{2} x_{1} x_{2}+x_{3}\right) & -\frac{1}{2} x_{2} \\
\frac{1}{2} x_{1} x_{2}+x_{3} & -1 & \frac{1}{2} x_{1} \\
\frac{1}{2} x_{2} & -\frac{1}{2} x_{1} & -2
\end{array}\right) .
$$

It follows that $2 \operatorname{Rc}(g)+\mathcal{L}_{X} g+3 g=0$, which verifies the Ricci soliton structure. Let $\xi$ denote the 1-form metrically dual to $X$. Recalling the standard identities

$$
\begin{aligned}
d \xi(V, W) & =V\langle X, W\rangle-W\langle X, V\rangle-\langle X,[V, W]\rangle \\
& =\left\langle\nabla_{V} X, W\right\rangle-\left\langle\nabla_{W} X, V\right\rangle,
\end{aligned}
$$

one observes that $\xi$ is not closed, hence that the soliton is nongradient.

The following observation confirms that we may study this soliton by the methods of Section 3 . 
Proposition 3. For any 'big bang' time $\beta<0$, the diffeomorphisms $\eta_{t}: \mathcal{M}^{3} \rightarrow \mathcal{M}^{3}$ defined by

$$
\eta_{t}\left(x_{1}, x_{2}, x_{3}\right)=\left(\left(\frac{\beta}{\beta-t}\right)^{1 / 3} x_{1},\left(\frac{\beta}{\beta-t}\right)^{1 / 3} x_{2},\left(\frac{\beta}{\beta-t}\right)^{2 / 3} x_{3}\right)
$$

exist for all $t>\beta$ and satisfy (3.1), with $\eta_{0}=\mathrm{id}$.

Proof. The result follows readily once one checks that in standard coordinates,

$$
X=-x_{1} \frac{\partial}{\partial x_{1}}-x_{2} \frac{\partial}{\partial x_{2}}-2 x_{3} \frac{\partial}{\partial x_{3}} .
$$

The relationship between the soliton structure $\left(\mathcal{M}^{3}, g, X, 3\right)$ and the evolving family of metrics whose existence is guaranteed by Theorem 2 is elucidated by the following.

Example 2. Consider the time-dependent frame $E(t):=F A(t)$, where

$$
A(t)=\left(\begin{array}{ccc}
0 & 0 & a(t) \\
0 & a(t) & 0 \\
-2 a^{2}(t) & 0 & 0
\end{array}\right) \quad \text { and } \quad a(t)=\sqrt{\frac{1}{12} t^{-2 / 3}} .
$$

With respect to $E(t)$, one obtains the identification

$$
\left(g_{i j}\right)_{E(t)}=\left(\begin{array}{ccc}
\frac{1}{9} t^{-4 / 3} & 0 & 0 \\
0 & \frac{1}{3} t^{-2 / 3} & 0 \\
0 & 0 & \frac{1}{3} t^{-2 / 3}
\end{array}\right) .
$$

Observe that we may identify $3 \operatorname{tg}_{E(t)}$ with Lott's limit solution [37, Formula (3.18)]

$$
g_{\infty}(t)=\frac{1}{3 t^{1 / 3}}\left(\theta^{1} \otimes \theta^{1}\right)+t^{1 / 3}\left(\theta^{2} \otimes \theta^{2}\right)+t^{1 / 3}\left(\theta^{3} \otimes \theta^{3}\right)
$$

given in coordinates $(x, y, z)$, with $\theta^{1}=d x+\frac{1}{2} y d z-\frac{1}{2} z d y, \theta^{2}=d y$, and $\theta^{3}=d z$.

Now let $h=h_{i j} \varphi^{i} \otimes \varphi^{j}$ be a smooth compactly supported tensor field. Let $H=\operatorname{tr}_{g} h$, noting that

and

$$
H=\frac{1}{4}\left(h_{11}+h_{22}+h_{33}\right)
$$

$$
|h|^{2}=\frac{1}{16}\left\{h_{11}^{2}+h_{22}^{2}+h_{33}^{2}+2\left(h_{12}^{2}+h_{13}^{2}+h_{23}^{2}\right)\right\}
$$

pointwise. Using Remark 2 in conjunction with the alternative formula

$$
\Delta_{\ell} h_{i j}=\Delta h_{i j}+\left(R h_{i j}+2 H R_{i j}\right)-3\left(R_{i}^{k} h_{k j}+R_{j}^{k} h_{i k}\right)+(2\langle\mathrm{Rc}, h\rangle-R H) g_{i j}
$$

for the Lichnerowicz Laplacian (3.3), valid when $n=3$, one computes that

$$
\begin{aligned}
\int\langle L h, h\rangle d \mu & =\int\langle\Delta h, h\rangle d \mu-\int\left\langle\nabla_{X} h, h\right\rangle d \mu \\
& +\int(R-\alpha)|h|^{2} d \mu+\int(4\langle\mathrm{Rc}, h\rangle-R H) H d \mu \\
& -6 \int R_{i}^{j} h_{j}^{k} h_{k}^{i} d \mu-2 \int \nabla_{i} X^{j} h_{j}^{k} h_{k}^{i} d \mu
\end{aligned}
$$

where all indices are with respect to the orthogonal frame field $F$. 
Clearly, $\int\langle\Delta h, h\rangle d \mu=-\|\nabla h\|^{2}$. Observing that $\delta X=4$, one integrates by parts to get

$$
-\int\left\langle\nabla_{X} h, h\right\rangle d \mu=-\frac{1}{2} \int(\delta X)\langle h, h\rangle d \mu=-2\|h\|^{2} .
$$

Because $R=-1 / 2$ and $\alpha=3$, one has $\int(R-\alpha)|h|^{2} d \mu=-\frac{7}{2}\|h\|^{2}$. The pointwise calculation $\langle\mathrm{Rc}, h\rangle=\frac{1}{8}\left(-h_{11}-h_{22}+h_{33}\right)$ shows that the fourth term reduces to

$$
\int(4\langle\mathrm{Rc}, h\rangle-R H) H d \mu=-\frac{3}{2}\|H\|^{2}+\int h_{33} H d \mu .
$$

Calculating pointwise that $R_{i}^{j} h_{j}^{k} h_{k}^{i}=\frac{1}{32}\left(-h_{11}^{2}-h_{22}^{2}+h_{33}^{2}-2 h_{12}^{2}\right)$, one gets

$$
-6 \int R_{i}^{j} h_{j}^{k} h_{k}^{i} d \mu=\frac{3}{16} \int\left(h_{11}^{2}+h_{22}^{2}-h_{33}^{2}+2 h_{12}^{2}\right) d \mu \text {. }
$$

Computing $\nabla_{i} X^{j} h_{j}^{k} h_{k}^{i}=-|h|^{2}-\frac{1}{16}\left(h_{33}^{2}+h_{13}^{2}+h_{23}^{2}\right)$ pointwise then gives

$$
-2 \int \nabla_{i} X^{j} h_{j}^{k} h_{k}^{i} d \mu=2\|h\|^{2}+\frac{1}{8} \int\left(h_{33}^{2}+h_{13}^{2}+h_{23}^{2}\right) d \mu .
$$

Putting these all together, one has

$\int\langle L h, h\rangle d \mu=-\|\nabla h\|^{2}-\frac{1}{2}\|h\|^{2}-\frac{3}{2}\|H\|^{2}+\int h_{33} H d \mu-\frac{1}{4} \int\left(h_{33}^{2}+h_{13}^{2}+h_{23}^{2}\right) d \mu$.

Since $\left|h_{33} H\right| \leq \frac{1}{4} h_{33}^{2}+H^{2}$ by weighted Cauchy-Schwarz, it follows that

$$
\int\langle L h, h\rangle d \mu \leq-\|\nabla h\|^{2}-\frac{1}{2}\left(\|h\|^{2}+\|H\|^{2}\right) .
$$

By [15, smooth compactly supported tensor fields are dense in $L^{2}$. Hence we have proved the following.

Proposition 4. The linearization of the modified Ricci flow (3.2) at the nil $^{3}$ soliton $\left(\mathcal{M}^{3}, g, X, 3\right)$ is strictly linearly stable and satisfies

$$
\int\langle L h, h\rangle d \mu \leq-\|\nabla h\|^{2}-\omega\|h\|^{2}
$$

with $\omega=1 / 2$.

4.2. sol $^{3}$ geometry. $\mathrm{A} \mathrm{sol}^{3}$ soliton $\left(\mathcal{M}^{3}, g, X, 4\right)$ has been found by Baird-Danielo [3] and independently by Lott [37, Section 3.3.2]. $\mathcal{M}^{3}$ is diffeomorphic to $\mathbb{R}^{3}$. In standard coordinates $\left(x_{1}, x_{2}, x_{3}\right)$, define a frame field $F=\left(F_{1}, F_{2}, F_{3}\right)$ by

$$
F_{1}=2 \frac{\partial}{\partial x_{1}}, F_{2}=2\left(e^{-x_{1}} \frac{\partial}{\partial x_{2}}+e^{x_{1}} \frac{\partial}{\partial x_{3}}\right), F_{3}=2\left(e^{-x_{1}} \frac{\partial}{\partial x_{2}}-e^{x_{1}} \frac{\partial}{\partial x_{3}}\right) .
$$

The bracket relations are $\left[F_{1}, F_{2}\right]=-2 F_{3},\left[F_{2}, F_{3}\right]=0$, and $\left[F_{3}, F_{1}\right]=2 F_{2}$. The connection is represented by

$$
\left(\nabla_{F_{i}} F_{j}\right)=\left(\begin{array}{ccc}
0 & 0 & 0 \\
2 F_{3} & 0 & -4 F_{1} \\
2 F_{2} & -4 F_{1} & 0
\end{array}\right)
$$

With respect to the dual field

$$
\varphi^{1}=\frac{1}{2} d x_{1}, \varphi^{2}=\frac{1}{4}\left(e^{x_{1}} d x_{2}+e^{-x_{1}} d x_{3}\right), \varphi^{3}=\frac{1}{4}\left(e^{x_{1}} d x_{2}-e^{-x_{1}} d x_{3}\right),
$$


we identify the metric $g=g_{i j} \varphi^{i} \otimes \varphi^{j}$ with the matrix

$$
\left(g_{i j}\right)=\left(\begin{array}{lll}
4 & 0 & 0 \\
0 & 8 & 0 \\
0 & 0 & 8
\end{array}\right)
$$

and its Ricci tensor $\mathrm{Rc}=R_{i j} \varphi^{i} \otimes \varphi^{j}$ with

$$
\left(R_{i j}\right)=\left(\begin{array}{ccc}
-8 & 0 & 0 \\
0 & 0 & 0 \\
0 & 0 & 0
\end{array}\right)
$$

Given any $\gamma \in \mathbb{R}$, define a vector field

$$
X=\gamma\left(-F_{1}-e^{-x_{1}} x_{3} F_{2}+e^{-x_{1}} x_{3} F_{3}\right)+(1-\gamma)\left(F_{1}-e^{x_{1}} x_{2} F_{2}-e^{x_{1}} x_{2} F_{3}\right) .
$$

A calculation shows that the components of $\nabla X=\nabla_{i} X^{j} \varphi^{i} \otimes F_{j}$ are

$$
\left(\nabla_{i} X^{j}\right)=\gamma\left(\begin{array}{ccc}
0 & 2 e^{-x_{1}} x_{3} & -2 e^{-x_{1}} x_{3} \\
-4 e^{-x_{1}} x_{3} & -2 & 0 \\
4 e^{-x_{1}} x_{3} & 0 & -2
\end{array}\right)+(1-\gamma)\left(\begin{array}{ccc}
0 & -2 e^{x_{1}} x_{2} & -2 e^{x_{1}} x_{2} \\
4 e^{x_{1}} x_{2} & -2 & 0 \\
4 e^{x_{1}} x_{2} & 0 & -2
\end{array}\right) .
$$

It follows that $2 \operatorname{Rc}(g)+\mathcal{L}_{X} g+4 g=0$, which verifies the Ricci soliton structure. As in Section 4.1, one observes that the soliton structure is nongradient.

Notice that in standard coordinates,

$$
X=\gamma\left(-2 \frac{\partial}{\partial x_{1}}-4 x_{3} \frac{\partial}{\partial x_{3}}\right)+(1-\gamma)\left(2 \frac{\partial}{\partial x_{1}}-4 x_{2} \frac{\partial}{\partial x_{2}}\right) .
$$

We verify the existence of suitable diffeomorphisms for the case $\gamma=1 / 2$, leaving the general case for an interested reader.

Proposition 5. Take $\gamma=1 / 2$ in (4.5). Then for any 'big bang' time $\beta<0$, the diffeomorphisms $\eta_{t}: \mathcal{M}^{3} \rightarrow \mathcal{M}^{3}$ defined by

$$
\eta_{t}\left(x_{1}, x_{2}, x_{3}\right)=\left(x_{1},\left(\frac{\beta}{\beta-t}\right)^{1 / 2} x_{2},\left(\frac{\beta}{\beta-t}\right)^{1 / 2} x_{3}\right)
$$

exist for all $t>\beta$ and satisfy (3.1), with $\eta_{0}=\mathrm{id}$.

The relationship between the soliton structure $\left(\mathcal{M}^{3}, g, X, 4\right)$ and the evolving family of metrics given by Theorem 2 can be seen as follows.

Example 3. Consider the time-dependent frame $E(t)=F A(t)$ given by

$$
A(t)=\left(\begin{array}{ccc}
0 & -\frac{1}{2} & 0 \\
a(t) & 0 & 0 \\
0 & 0 & a(t)
\end{array}\right) \quad \text { and } \quad a(t)=\sqrt{\frac{1}{32} t} .
$$

With respect to the frame $E(t)$, one has the identification

$$
\left(g_{i j}\right)_{E(t)}=\left(\begin{array}{ccc}
\frac{1}{4} t^{-1} & 0 & 0 \\
0 & 1 & 0 \\
0 & 0 & \frac{1}{4} t^{-1}
\end{array}\right) .
$$

Observe that we may identify $4 t g_{E(t)}$ with Lott's limit solution [37, Formula (3.9)]

$$
g_{\infty}(t)=\left(\theta^{1} \otimes \theta^{1}\right)+4 t\left(\theta^{2} \otimes \theta^{2}\right)+\left(\theta^{3} \otimes \theta^{3}\right)
$$

given in coordinates $(x, y, z)$, with $\theta^{1}+\theta^{3}=e^{-z} d x, \theta^{1}-\theta^{3}=e^{z} d y$, and $\theta^{2}=d z$. 
Now let $h=h_{i j} \varphi^{i} \otimes \varphi^{j}$ be a smooth compactly supported tensor field. Let $H=\operatorname{tr}_{g} h$, noting that

$$
H=\frac{1}{8}\left(2 h_{11}+h_{22}+h_{33}\right)
$$

and

$$
|h|^{2}=\frac{1}{64}\left\{4 h_{11}^{2}+h_{22}^{2}+h_{33}^{2}+4 h_{12}^{2}+4 h_{13}^{2}+2 h_{23}^{2}\right\}
$$

pointwise. As in Section 4.1, we proceed to evaluate

$$
\begin{aligned}
\int\langle L h, h\rangle d \mu & =\int\langle\Delta h, h\rangle d \mu-\int\left\langle\nabla_{X} h, h\right\rangle d \mu \\
& +\int(R-\alpha)|h|^{2} d \mu+\int(4\langle\mathrm{Rc}, h\rangle-R H) H d \mu \\
& -6 \int\left\langle\mathrm{Rc}, h^{2}\right\rangle d \mu-2 \int \nabla_{i} X^{j} h_{j}^{k} h_{k}^{i} d \mu .
\end{aligned}
$$

Since $\alpha=4=\delta X, R=-2,\langle\mathrm{Rc}, h\rangle=-\frac{1}{2} h_{11}$, and $\left\langle\mathrm{Rc}, h^{2}\right\rangle=-\frac{1}{16}\left(2 h_{11}^{2}+h_{12}^{2}+h_{13}^{2}\right)$, one finds that

$$
\int\langle L h, h\rangle d \mu=-\|\nabla h\|^{2}-\frac{1}{32} \int P d \mu
$$

where

$$
P:=32 H h_{11}-4 h_{11}^{2}+\left(h_{22}-h_{33}\right)^{2}+4 h_{23}^{2} .
$$

At a point where $h=\operatorname{diag}(a,-2 a,-2 a)$, one has $\frac{1}{32} P=-\frac{3}{8} a^{2}=-2|h|^{2}$, so we must work harder.

As in [33], one defines a $(3,0)$-tensor $T=T(h)$ by

$$
T_{i j k}:=\nabla_{k} h_{i j}-\nabla_{i} h_{j k}
$$

and computes that

$$
\|\nabla h\|^{2}=\|\delta h\|^{2}+\frac{1}{2}\|T\|^{2}+\int\left\{R_{i j k \ell} h^{i \ell} h^{j k}-R_{i}^{k} h_{j k} h^{i j}\right\} d \mu .
$$

The decomposition of the Riemann curvature tensor in dimension three,

$$
R_{i j k \ell}=R_{i \ell} g_{j k}+R_{j k} g_{i \ell}-R_{i k} g_{j \ell}-R_{j \ell} g_{i k}-\frac{1}{2} R\left(g_{i \ell} g_{j k}-g_{i k} g_{j \ell}\right),
$$

implies the pointwise identity

$$
R_{i j k \ell} h^{i \ell} h^{j k}-R_{i}^{k} h_{j k} h^{i j}=2\langle\mathrm{Rc}, h\rangle H-3\left\langle\mathrm{Rc}, h^{2}\right\rangle-\frac{1}{2} R H^{2}+\frac{1}{2} R|h|^{2} .
$$

Using this and discarding the $\|T\|^{2}$ term, one can improve (4.6), obtaining

$$
\int\langle L h, h\rangle d \mu \leq-\|\delta h\|^{2}-\frac{1}{64} \int Q d \mu,
$$

where

$$
Q:=16 h_{11}^{2}+h_{22}^{2}+h_{33}^{2}+8 h_{12}^{2}+8 h_{13}^{2}+6 h_{23}^{2}+\left(h_{22}-h_{33}\right)^{2}+4 h_{11}\left(h_{22}+h_{33}\right) .
$$

Recall that $64|h|^{2}=4 h_{11}^{2}+h_{22}^{2}+h_{33}^{2}+4 h_{12}^{2}+4 h_{13}^{2}+2 h_{23}^{2}$. Recall too that for any $\varepsilon>0$, one has $\left|4 h_{11}\left(h_{22}+h_{33}\right)\right| \leq 4 \varepsilon h_{11}^{2}+\frac{2}{\varepsilon}\left(h_{22}^{2}+h_{33}^{2}\right)$. The choice $\varepsilon=(3+\sqrt{17}) / 2$ solves $(16-4 \varepsilon) / 4=1-2 / \varepsilon$, hence is optimal. Thus we obtain the following result. 
Proposition 6. The linearization of the modified Ricci flow (3.2) at the $\mathrm{sol}^{3}$ soliton $\left(\mathcal{M}^{3}, g, X, 4\right)$ is strictly linearly stable and satisfies

$$
\int\langle L h, h\rangle d \mu \leq-\|\delta h\|^{2}-\omega\|h\|^{2}
$$

with $\omega=(5-\sqrt{17}) / 2>0$.

4.3. nil ${ }^{4}$ geometry. We now describe the soliton $\left(\mathcal{M}^{4}, g, X, 3\right)$ introduced in [37, Section 3.4.9]. (Compare [28, Section A.6].) In standard coordinates $\left(x_{1}, x_{2}, x_{3}, x_{4}\right)$ on $\mathcal{M}^{4} \approx \mathbb{R}^{4}$, consider the frame field

$$
F_{1}=\frac{\partial}{\partial x_{1}}, F_{2}=\frac{\partial}{\partial x_{2}}, F_{3}=\frac{\partial}{\partial x_{3}}, F_{4}=x_{1} \frac{\partial}{\partial x_{2}}+x_{2} \frac{\partial}{\partial x_{3}}+\frac{\partial}{\partial x_{4}} .
$$

It is easy to check that $\left[F_{1}, F_{4}\right]=F_{2}$ and $\left[F_{2}, F_{4}\right]=F_{3}$ with all other brackets vanishing. The dual basis is

$$
\varphi^{1}=d x_{1}, \varphi^{2}=d x_{2}-x_{1} d x_{4}, \varphi^{3}=d x_{3}-x_{2} d x_{4}, \varphi^{4}=d x_{4},
$$

and the connection is specified by

$$
\left(\nabla_{F_{i}} F_{j}\right)=\frac{1}{2}\left(\begin{array}{cccc}
0 & -F_{4} & 0 & F_{2} \\
-F_{4} & 0 & -F_{4} & F_{1}+F_{3} \\
0 & -F_{4} & 0 & F_{2} \\
-F_{2} & F_{1}-F_{3} & F_{2} & 0
\end{array}\right)
$$

Let $g=g_{i j} \varphi^{i} \otimes \varphi^{j}$ denote Lott's metric $g_{\infty}\left(\frac{1}{3}\right)$, where by [37, Formula (3.66)],

$g_{\infty}(t)=3^{1 / 3} t^{1 / 3}\left(\varphi^{1} \otimes \varphi^{1}\right)+\left(\varphi^{2} \otimes \varphi^{2}\right)+3^{-1 / 3} t^{-1 / 3}\left(\varphi^{3} \otimes \varphi^{3}\right)+3^{2 / 3} t^{2 / 4}\left(\varphi^{4} \otimes \varphi^{4}\right)$.

Then $g$ corresponds to the matrix

$$
g=\left(\begin{array}{llll}
1 & 0 & 0 & 0 \\
0 & 1 & 0 & 0 \\
0 & 0 & 1 & 0 \\
0 & 0 & 0 & 1
\end{array}\right),
$$

and its Ricci curvature tensor $\mathrm{Rc}=R_{i j} \varphi^{i} \otimes \varphi^{j}$ corresponds to

$$
\mathrm{Rc}=\left(\begin{array}{cccc}
-1 / 2 & 0 & 0 & 0 \\
0 & 0 & 0 & 0 \\
0 & 0 & 1 / 2 & 0 \\
0 & 0 & 0 & 1
\end{array}\right)
$$

Consider the vector field

$$
X=-2 x_{1} F_{1}+\left(-3 x_{2}+x_{1} x_{4}\right) F_{2}+\left(-4 x_{3}+x_{2} x_{4}\right) F_{3}-x_{4} F_{4} .
$$

Calculating

$$
\left(\nabla_{i} X^{j}\right)=\left(\begin{array}{cccc}
-2 & \frac{1}{2} x_{4} & 0 & \frac{1}{2}\left(3 x_{2}-x_{1} x_{4}\right) \\
-\frac{1}{2} x_{4} & -3 & \frac{1}{2} x_{4} & x_{1}+2 x_{3}-\frac{1}{2} x_{2} x_{4} \\
0 & -\frac{1}{2} x_{4} & -4 & \frac{1}{2}\left(3 x_{2}-x_{1} x_{4}\right) \\
\frac{1}{2}\left(x_{1} x_{4}-3 x_{2}\right) & \frac{1}{2} x_{2} x_{4}-x_{1}-2 x_{3} & \frac{1}{2}\left(x_{1} x_{4}-3 x_{2}\right) & -1
\end{array}\right),
$$

we have verified the nongradient Ricci soliton structure $2 \operatorname{Rc}(g)+\mathcal{L}_{X} g+3 g=0$. 
Proposition 7. For any 'big bang' time $\beta<0$, the diffeomorphisms $\eta_{t}: \mathcal{M}^{4} \rightarrow \mathcal{M}^{4}$ defined by

$$
\eta_{t}\left(x_{1}, x_{2}, x_{3}, x_{4}\right)=\left(\left(\frac{\beta}{\beta-t}\right)^{2 / 3} x_{1},\left(\frac{\beta}{\beta-t}\right) x_{2},\left(\frac{\beta}{\beta-t}\right)^{4 / 3} x_{3},\left(\frac{\beta}{\beta-t}\right)^{1 / 3} x_{4}\right)
$$

exist for all $t>\beta$ and satisfy (3.1), with $\eta_{0}=\mathrm{id}$.

Proof. The result follows immediately from the observation that in standard coordinates,

$$
X=-2 x_{1} \frac{\partial}{\partial x_{1}}-3 x_{2} \frac{\partial}{\partial x_{2}}-4 x_{3} \frac{\partial}{\partial x_{3}}-x_{4} \frac{\partial}{\partial x_{4}} .
$$

Let $h=h_{i j} \varphi^{i} \otimes \varphi^{j}$ be a smooth compactly supported tensor field. By formulas (3.5) and (3.3), one has

$$
\begin{aligned}
\int\langle L h, h\rangle d \mu & =\int\langle\Delta h, h\rangle d \mu-\int\left\langle\nabla_{X} h, h\right\rangle d \mu \\
& +2 \int R_{i j k \ell} h^{i \ell} h^{j k} d \mu-2 \int R_{i}^{j} h_{j}^{k} h_{k}^{i} d \mu \\
& -3\|h\|^{2}-2 \int \nabla_{i} X^{j} h_{j}^{k} h_{k}^{i} d \mu,
\end{aligned}
$$

where all indices are with respect to the orthonormal frame $F$. Since formula (4.3) is not available, one must calculate the full curvature tensor to proceed. So define $R_{i j k}:=R\left(F_{i}, F_{j}\right) F_{k}=\nabla_{F_{i}}\left(\nabla_{F_{j}} F_{k}\right)-\nabla_{F_{j}}\left(\nabla_{F_{i}} F_{k}\right)-\nabla_{\left[F_{i}, F_{j}\right]} F_{k}$. Then it is straightforward to compute that all nonvanishing curvature components are determined by

$$
\begin{aligned}
\frac{1}{4} F_{2} & =R_{211}=R_{213}=R_{231}=R_{233}=\frac{1}{2} R_{424} \\
\frac{1}{4} F_{4} & =\frac{1}{3} R_{141}=R_{143}=\frac{1}{2} R_{242}=R_{341}=R_{433} \\
\frac{1}{4} F_{1}+\frac{1}{4} F_{3} & =R_{122}=R_{322} \\
\frac{1}{4} F_{1}-\frac{1}{4} F_{3} & =R_{434} \\
\frac{3}{4} F_{1}+\frac{1}{4} F_{3} & =R_{414} .
\end{aligned}
$$

Further pointwise calculations show that

$$
\begin{aligned}
2 R_{i j k \ell} h^{i \ell} h^{j k} & =-h_{12}^{2}+3 h_{14}^{2}-h_{23}^{2}+2 h_{24}^{2}-h_{34}^{2} \\
& +h_{11} h_{22}-3 h_{11} h_{44}-2 h_{12} h_{23}+2 h_{13} h_{22}-2 h_{13} h_{44} \\
& +2 h_{14} h_{34}+h_{22} h_{33}-2 h_{22} h_{44}+h_{33} h_{44}
\end{aligned}
$$

and

$$
2 R_{i}^{j} h_{j}^{k} h_{k}^{i}=-h_{11}^{2}+h_{33}^{2}-2 h_{44}^{2}-h_{12}^{2}-3 h_{14}^{2}+h_{23}^{2}-2 h_{24}^{2}-h_{34}^{2}
$$

and

$$
\begin{aligned}
\nabla_{i} X^{j} h_{j}^{k} h_{k}^{i} & =-2 h_{11}^{2}-3 h_{22}^{2}-4 h_{33}^{2}-h_{44}^{2} \\
& -5 h_{12}^{2}-6 h_{13}^{2}-3 h_{14}^{2}-4 h_{24}^{2}-7 h_{23}^{2}-5 h_{34}^{2} .
\end{aligned}
$$


Because $\delta X=10$, one has $-\int\left\langle\nabla_{X} h, h\right\rangle d \mu=-5\|h\|^{2}$. Collecting terms, one then concludes that

$$
\int\langle L h, h\rangle d \mu=-\|\nabla h\|^{2}+\int Q d \mu
$$

where

$$
\begin{aligned}
Q & :=-3 h_{11}^{2}-2 h_{22}^{2}-h_{33}^{2}-4 h_{44}^{2}-6 h_{12}^{2}-4 h_{13}^{2}-4 h_{14}^{2}-4 h_{23}^{2}-4 h_{24}^{2}-6 h_{34}^{2} \\
& +h_{11} h_{22}-3 h_{11} h_{44}-2 h_{12} h_{23}+2 h_{13} h_{22}-2 h_{13} h_{44} \\
& +2 h_{14} h_{34}+h_{22} h_{33}-2 h_{22} h_{44}+h_{33} h_{44} .
\end{aligned}
$$

Proposition 8. The linearization of the modified Ricci flow (3.2) at the nil ${ }^{4}$ soliton $\left(\mathcal{M}^{4}, g, X, 3\right)$ is strictly linearly stable and satisfies

$$
\int\langle L h, h\rangle d \mu \leq-\|\nabla h\|^{2}-\omega\|h\|^{2}
$$

for some $\omega>0.0057$.

Proof. For constants $A, B, C, D, E, F>0$ to be chosen, weighted Cauchy-Schwarz implies that $\left|h_{11} h_{22}\right| \leq \frac{1}{4 A} h_{11}^{2}+A h_{22}^{2}, 2\left|h_{13} h_{22}\right| \leq \frac{1}{2 B} h_{13}^{2}+2 B h_{22}^{2}, 2\left|h_{13} h_{44}\right| \leq$ $\frac{1}{2 C} h_{13}^{2}+2 C h_{44}^{2},\left|h_{22} h_{33}\right| \leq D h_{22}^{2}+\frac{1}{4 D} h_{33}^{2}, 2\left|h_{22} h_{44}\right| \leq 2 E h_{22}^{2}+\frac{1}{2 E} h_{44}^{2}$, and $\left|h_{33} h_{44}\right| \leq$ $F h_{33}^{2}+\frac{1}{4 F} h_{44}^{2}$. This leads to the estimate

$$
\begin{aligned}
Q & \leq\left(-\frac{3}{2}+\frac{1}{4 A}\right) h_{11}^{2}+(-2+A+2 B+D+2 E) h_{22}^{2} \\
& +\left(-1+\frac{1}{4 D}+F\right) h_{33}^{2}+\left(-\frac{5}{2}+2 C+\frac{1}{2 E}+\frac{1}{4 F}\right) h_{44}^{2} \\
& -5 h_{12}^{2}+\left(-4+\frac{1}{2 B}+\frac{1}{2 C}\right) h_{13}^{2}-3 h_{14}^{2}-3 h_{23}^{2}-4 h_{24}^{2}-5 h_{34}^{2} .
\end{aligned}
$$

The choices $A=0.17, B=0.27, C=0.24, D=0.35, E=0.46$, and $F=0.28$ (which are not optimal!) yield the estimate $Q \leq-\omega|h|^{2}$.

\section{Generation of Strongly Continuous Semigroups}

Our approach in this section incorporates ideas of Rabier [4], adapting them to tensor-valued operators on noncompact manifolds. Roughly speaking, the idea is to approximate an unbounded operator by a sequence of bounded operators, obtain uniform estimates for this sequence, and then use the fact that all but finitely many of the approximants agree with the original operator when paired against any tensor field with compact support.

Let $\left(\mathcal{M}^{n}, g, X, \alpha\right)$ be the $\mathrm{nil}^{3}$, $\mathrm{sol}^{3}$, or nil ${ }^{4}$ soliton discussed above. For each $i, j, k \in \mathbb{N}$, we denote by $W_{i, j}^{k} \equiv W_{i, j}^{k, 2}$ the Sobolev space of complex-valued $(i, j)$ tensor fields on $\mathcal{M}^{n}$, obtained as the completion of $C_{0}^{\infty}\left(T_{i}^{j} \mathcal{M}^{n} ; \mathbb{C}\right)$ with respect to the norm

$$
\|u\|_{k}^{2}=\sum_{\ell=0}^{k} \int_{\mathcal{M}^{n}}\left|\nabla^{\ell} u\right|^{2} d \mu=\sum_{\ell=0}^{k}\left(\nabla^{\ell} u, \nabla^{\ell} u\right) .
$$

To avoid notational prolixity, we write each $L_{i, j}^{2}=W_{i, j}^{0}$ inner product as $(\cdot, \cdot)$; the relevant values of $i, j$ should be clear from the context. We denote the real and imaginary parts of a complex number $z$ by $\operatorname{Re}(z)$ and $\operatorname{Im}(z)$, respectively. 
Lemma 1. Let $\xi \in W_{0,1}^{1}$ be a real vector field with $\|\delta \xi\|_{\infty}<\infty$, and let $\zeta$ be a real $(2,2)$-tensor field with $\|\zeta\|_{\infty}<\infty$. Assume there exists $\omega \in \mathbb{R}$ such that for all $v \in W_{2,0}^{1}$, one has

$$
\left(\frac{1}{2}(\delta \xi) v-\zeta v, \bar{v}\right) \geq \omega\|v\|_{0}^{2} .
$$

Then for each $\lambda>-\omega$ and each $f \in L_{2,0}^{2}$, there exists a unique $u \in W_{2,0}^{1}$ with norm

$$
\|u\|_{1}^{2} \leq \frac{1}{\lambda+\omega}\|f\|_{0}^{2}
$$

such that $u$ solves $\beta_{\lambda}(u, v)=(f, \bar{v})$ for all $v \in W_{2,0}^{1}$, where

$$
\beta_{\lambda}(u, v):=\lambda(u, \bar{v})+(\nabla u, \nabla \bar{v})+\left(\nabla_{\xi} u, \bar{v}\right)-(\zeta u, \bar{v}) .
$$

Proof. For each $\lambda \in \mathbb{R}, \beta_{\lambda}$ is a continuous sesquilinear form on $W_{2,0}^{1}$. Fix $\lambda>-\omega$. By assumption (5.1), the fact that smooth compactly-supported tensor fields are dense in $W_{2,0}^{1}$ implies that for all $u \in W_{2,0}^{1}$, one can integrate by parts to get

$$
\operatorname{Re}\left(\beta_{\lambda}(u, u)\right) \geq\|\nabla u\|_{0}^{2}+(\lambda+\omega)\|u\|_{0}^{2} \geq \min \{1, \lambda+\omega\}\|u\|_{1}^{2} .
$$

By the Lax-Milgram Theorem, for each $f \in L_{2,0}^{2}$, there exists a unique $u \in W_{2,0}^{1}$ solving $\beta_{\lambda}(u, v)=(f, \bar{v})$ for all $v \in W_{2,0}^{1}$ and satisfying the estimate

$$
\|u\|_{1} \leq \max \left\{1,(\lambda+\omega)^{-1}\right\}\|f\|_{0} .
$$

It follows that $(\lambda+\omega)\|u\|_{0}^{2} \leq \operatorname{Re}\left(\beta_{\lambda}(u, u)\right)=\operatorname{Re}(f, \bar{u}) \leq\|f\|_{0}\|u\|_{0}$, which implies that

$$
\|u\|_{0} \leq \frac{1}{\lambda+\omega}\|f\|_{0}
$$

and hence that

$$
\|\nabla u\|_{0}^{2}+(\lambda+\omega)\|u\|_{0}^{2} \leq \operatorname{Re}((f, \bar{u})) \leq \frac{1}{\lambda+\omega}\|f\|_{0}^{2} .
$$

Since the left-hand side of (5.4) is an upper bound for $\|u\|_{1}^{2}$ when $\lambda+\omega \geq 1$, the conclusion follows from (5.3) and (5.4).

Recall the linear operator $L$ defined by (3.4). By Remark 2, $L$ can be written in the form

$$
L h=\Delta h-\nabla_{X} h+Z h,
$$

where $Z$ is an unbounded $(2,2)$-tensor field. For each soliton under consideration, our calculations in Section 3 show that $\delta X$ is constant. Moreover, by Propositions 4 . [6, and 8, there exists $\omega>0$ depending only on the soliton in question such that

$$
\left\langle\frac{1}{2}(\delta X) v-Z v, v\right\rangle \geq \omega|v|^{2}
$$

holds pointwise for all real compactly supported $(2,0)$-tensor fields $v$.

Let $r=\sqrt{\sum_{\ell=1}^{n} x_{\ell}^{2}}$ denote the Euclidean distance from the origin in $\mathbb{R}^{n} \approx \mathcal{M}^{n}$. By formulas (4.2), (4.5), and (4.8), there exists $C>0$ depending only on the Lie group in question such that

$$
d r(X) \geq-C r .
$$


For each $k=1,2, \ldots$, let $\gamma_{k}$ be a translated smooth bump function such that $\gamma_{k}(s)=1$ for $s<k-1+\varepsilon, \gamma_{k}(s)=0$ for $s>k-\varepsilon$, and $-2 \leq \gamma^{\prime}(s) \leq 0$ for all $s$. Define the vector fields

$$
\xi_{k}(x):=\gamma_{k}(r(x)) X(x),
$$

noting that $\xi_{k}$ is supported in the Euclidean ball of radius $k$. Define

$$
\zeta_{k}(x):=\left\{\begin{array}{cl}
Z(x) & \text { if } r(x)<k-1 \\
\gamma_{k}(r(x)) Z(x)-(C k+\omega) & \text { if } r(x) \geq k-1
\end{array},\right.
$$

where $C$ is given by (5.6). Note that $\zeta_{k}$ is smooth except on the hypersurface $r=k-1$.

Lemma 2. Assume (5.5) and (5.6) hold. Fix any $\lambda>-\omega$ and let $f \in L_{2,0}^{2}$ be given. Then for each integer $k \geq 1$, there exists a unique $u_{k} \in W_{2,0}^{1}$ solving

$$
\lambda\left(u_{k}, \bar{v}\right)+\left(\nabla u_{k}, \nabla \bar{v}\right)+\left(\nabla_{\xi_{k}} u_{k}, \bar{v}\right)-\left(\zeta_{k} u_{k}, \bar{v}\right)=(f, \bar{v})
$$

for all $v \in W_{2,0}^{1}$, where $\xi_{k}$ and $\zeta_{k}$ are defined in (5.7) and (5.8), respectively. Furthermore, all $u_{k}$ are bounded uniformly by

$$
\left\|u_{k}\right\|_{1}^{2} \leq \frac{1}{\lambda+\omega}\|f\|_{0}^{2}
$$

Proof. If $r<k-1$, then (5.5) gives the pointwise inequality

$$
\left\langle\frac{1}{2}\left(\delta \xi_{k}\right) v-\zeta_{k} v, v\right\rangle=\left\langle\frac{1}{2}(\delta X) v-Z v, v\right\rangle \geq \omega|v|^{2}
$$

for all real $v$. If $r \geq k-1$, then we have

$$
\left\langle\frac{1}{2}\left(\delta \xi_{k}\right) v-\zeta_{k} v, v\right\rangle \geq \gamma_{k}(r)\left\langle\frac{1}{2}(\delta X) v-Z v, v\right\rangle+\omega|v|^{2} \geq \omega|v|^{2}
$$

for all real $v$ by (5.5), (5.6), the fact that $\xi_{k}$ is supported in $r<k$, and our choice of $\zeta_{k}$. Applying Lemma 1 with $\xi=\xi_{k}$ and $\zeta=\zeta_{k}$ thus yields the result.

Now let $\mathbb{L}$ denote the complexification of the linear operator $L$ defined by (3.4). $\mathbb{L}$ is defined for all $u=v+i w$ (with $v, w$ real) by

$$
\mathbb{L} u:=L v+i L w .
$$

A priori, $\mathbb{L}$ is only defined on $C_{0}^{\infty}\left(T_{2}^{0} \mathcal{M}^{n} ; \mathbb{C}\right)$.

Lemma 3. Assume (5.5) and (5.6) hold. Fix any $\lambda>-\omega$. Then for each $f \in L_{2,0}^{2}$, there exists a weak solution $u \in W_{2,0}^{1}$ of

$$
(\lambda \mathbb{I}-\mathbb{L}) u=f
$$

such that

$$
\|u\|_{1}^{2} \leq \frac{1}{\lambda+\omega}\|f\|_{0}^{2} .
$$

Proof. Let $\left\{u_{k}\right\}_{k \geq 1}$ be the sequence given by Lemma 2. Since $u_{k}$ is bounded uniformly in $W_{2,0}^{1}$, a subsequence $\left\{u_{k_{j}}\right\}_{j \in \mathbb{N}}$ converges weakly to some $u \in W_{2,0}^{1}$. Thus the $(\lambda \mathbb{I}-\mathbb{L}) u_{k_{j}}$ converge to $(\lambda \mathbb{I}-\mathbb{L}) u$ as distributions. This means that for any smooth compactly-supported $(2,0)$-tensor field $v$, one has

$$
\lim _{j \rightarrow \infty}\left((\lambda \mathbb{I}-\mathbb{L}) u_{k_{j}}, \bar{v}\right)=((\lambda \mathbb{I}-\mathbb{L}) u, \bar{v}),
$$

where we make sense of the Laplacian term by integration by parts. 
Recall that $\xi_{k}=X$ and $\zeta_{k}=Z$ wherever $r<k-1$. Because the support $\operatorname{Sprt}(v)$ of $v$ is compact, there exists $J$ depending on $v$ such that

$$
\begin{aligned}
\left((\lambda \mathbb{I}-\mathbb{L}) u_{k_{j}}, \bar{v}\right) & =\lambda\left(u_{k_{j}}, \bar{v}\right)+\left(\nabla u_{k_{j}}, \nabla \bar{v}\right)+\left(\nabla_{X} u_{k_{j}}, \bar{v}\right)-\left(Z u_{k_{j}}, \bar{v}\right) \\
& =\lambda\left(u_{k_{j}}, \bar{v}\right)+\left(\nabla u_{k_{j}}, \nabla \bar{v}\right)+\left(\nabla_{\xi_{k_{j}}} u_{k_{j}}, \bar{v}\right)-\left(\zeta_{k_{j}} u_{k_{j}}, \bar{v}\right) \\
& =(f, \bar{v})
\end{aligned}
$$

for all $j \geq J$. The first equality above follows directly from the definition of $\mathbb{L}$. The second equality holds because $\left.\xi_{k_{j}}\right|_{\operatorname{Sprt}(v)}=\left.X\right|_{\operatorname{Sprt}(v)}$ and $\left.\zeta_{k_{j}}\right|_{\operatorname{Sprt}(v)}=\left.Z\right|_{\operatorname{Sprt}(v)}$ for all $j \geq J$, by our choice of $J$. The last equality is (5.9). Combining these observations, we see that $((\lambda \mathbb{I}-\mathbb{L}) u, \bar{v})=(f, \bar{v})$ for all compactly supported $v$. By density of smooth compactly-supported tensor fields, this is possible only if $(\lambda \mathbb{I}-\mathbb{L}) u=f$. Since weak convergence $u_{k_{j}} \rightarrow u$ implies that

$$
\|u\|_{1} \leq \liminf _{j \rightarrow \infty}\left\|u_{k_{j}}\right\|_{1}
$$

the result follows.

Now for any $\lambda>-\omega$, we may define the domain of $\mathbb{L}$ by

$$
\mathrm{D}_{\mathbb{L}}=(\lambda \mathbb{I}-\mathbb{L})^{-1}\left(L_{2,0}^{2}\right) .
$$

By the resolvent identity $(\mu \mathbb{I}-\mathbb{L})^{-1}=(\lambda \mathbb{I}-\mathbb{L})^{-1}+(\lambda-\mu)(\lambda \mathbb{I}-\mathbb{L})^{-1}(\mu \mathbb{I}-\mathbb{L})^{-1}, \mathrm{D}_{\mathbb{L}}$ is well defined, i.e. independent of $\lambda>-\omega$. Because it contains $C_{0}^{\infty}\left(T_{2}^{0} \mathcal{M}^{n} ; \mathbb{C}\right)$, the domain $\mathrm{D}_{\mathbb{L}}$ is dense in each $W_{2,0}^{k}$. The following is then an immediate consequence of Lemma 3 and the Hille-Yosida Theorem. (See [40, Theorem 3.1].)

Theorem 5. $\mathbb{L}$ generates a unique $C_{0}$ semigroup with domain $\mathrm{D}_{\mathbb{L}}$. In particular, for every $f \in L_{2,0}^{2}$ and $\lambda \in \mathbb{C}$ with $\operatorname{Re}(\lambda)>-\omega$, there exists $u \in W_{2,0}^{1}$ solving $u=(\lambda \mathbb{I}-\mathbb{L})^{-1} f$ and satisfying

$$
\|u\|_{1}^{2} \leq \frac{1}{\operatorname{Re}(\lambda)+\omega}\|f\|_{0}^{2} .
$$

\section{Directions For Further RESEARCH}

This note is a contribution to a challenging program to investigate analytic aspects of large-time behavior of immortal (especially Type-III) Ricci flow solutions, and in particular to investigate Conjectures 1 and 2, One aspect of this program may be divided into three main parts, all of which are undergoing active development:

(1) Catalog examples of expanding homogeneous Ricci solitons as models of Type-III behavior.

(2) Determine asymptotic stability of these homogeneous Ricci solitons.

(3) Deduce analytic properties of their pseudosoliton quotients.

Contributions to Part 1 have been made by Lauret [34, Lauret-Will [35, BairdDanielo [3, and Lott [37, among others. Results of Glickenstein [18] and Lott [37] contribute to Part 3, as do the theories of Riemannian groupoids and megafolds. (See 21] and [43, for example.) Part 2 is still largely open. We hope this note stimulates progress toward its resolution. 


\section{REFERENCES}

[1] Angenent, Sigurd B.; Knopf, Dan. An example of neckpinching for Ricci flow on $S^{n+1}$. Math. Res. Lett. 11 (2004), no. 4, 493-518.

[2] Angenent, Sigurd B.; Knopf, Dan. Precise asymptotics for the Ricci flow neckpinch. arXiv:math.DG/0511247.

[3] Baird, Paul; Danielo, Laurent. Three-dimensional Ricci solitons which project to surfaces. arXiv:math.DG/0510313

[4] Berndt, Jürgen; Tricerri, Franco; Vanhecke, Lieven. Generalized Heisenberg groups and Damek-Ricci harmonic spaces. Lecture Notes in Mathematics, 1598. Springer-Verlag, Berlin, 1995.

[5] Biquard, Olivier. Métriques d'Einstein asymptotiquement symétriques. Astérisque No. 265 (2000).

[6] Cheeger, Jeff; Fukaya, Kenji; Gromov, Mikhael. Nilpotent structures and invariant metrics on collapsed manifolds. J. Amer. Math. Soc. 5 (1992), no. 2, 327-372.

[7] Cheeger, Jeff; Gromov, Mikhael. Collapsing Riemannian manifolds while keeping their curvature bounded. I. J. Differential Geom. 23 (1986), no. 3, 309-346.

[8] Cheeger, Jeff; Gromov, Mikhael. Collapsing Riemannian manifolds while keeping their curvature bounded. II. J. Differential Geom. 32 (1990), no. 1, 269-298.

[9] Cupini, Giovanni; Fornaro, Simona. Maximal regularity in $L^{p}\left(\mathbb{R}^{N}\right)$ for a class of elliptic operators with unbounded coefficients. Differential Integral Equations 17 (2004), no. 3-4, 259-296.

[10] Cannarsa, Piermarco; Vespri, Vincenzo. Generation of analytic semigroups by elliptic operators with unbounded coefficients. SIAM J. Math. Anal. 18 (1987), no. 3, 857-872.

[11] Cao, Huai-Dong; Zhu, Xi-Ping. A Complete Proof of the Poincaré and Geometrization Conjectures - application of the Hamilton-Perelman theory of the Ricci flow. Asian J. Math. 10 (2006), no. 2, 165-492.

[12] Da Prato, G.; Lunardi, A. Stability, instability and center manifold theorem for fully nonlinear autonomous parabolic equations in Banach space. Arch. Rational Mech. Anal. 101 (1988), no. 2, 115-141.

[13] DeTurck, Dennis M. Deforming metrics in the direction of their Ricci tensors. J. Differential Geom. 18 (1983), no. 1, 157-162.

[14] DeTurck, Dennis M. Deforming metrics in the direction of their Ricci tensors, improved version. Collected Papers on Ricci Flow. Edited by H.-D. Cao, B. Chow, S.-C. Chu, and S.-T. Yau. Internat. Press, Somerville, MA, 2003.

[15] Eichhorn, Jürgen; Fricke, Jan. The module structure theorem for Sobolev spaces on open manifolds. Math. Nachr. 194 (1998), 35-47.

[16] Fukaya, Kenji. Collapsing Riemannian manifolds to ones of lower dimensions. J. Differential Geom. 25 (1987), no. 1, 139-156.

[17] Fukaya, Kenji. Collapsing Riemannian manifolds to ones with lower dimension. II. J. Math. Soc. Japan 41 (1989), no. 2, 333-356.

[18] Glickenstein, David. Precompactness of solutions to the Ricci flow in the absence of injectivity radius estimates. Geom. Topol. 7 (2003), 487-510.

[19] Guenther, Christine; Isenberg, James; Knopf, Dan. Stability of the Ricci flow at Ricci-flat metrics. Comm. Anal. Geom. 10 (2002), no. 4, 741-777.

[20] Gozzi, Fausto; Monte, Roberto; Vespri, Vincenzo. Generation of analytic semigroups and domain characterization for degenerate elliptic operators with unbounded coefficients arising in financial mathematics. I. Differential Integral Equations 15 (2002), no. 9, 10851128.

[21] Haefliger, André. Groupoids and foliations. Groupoids in analysis, geometry, and physics (Boulder, CO, 1999), 83-100, Contemp. Math., 282, Amer. Math. Soc., Providence, RI, 2001.

[22] Hamilton, Richard S. The formation of singularities in the Ricci flow. Surveys in differential geometry, Vol. II (Cambridge, MA, 1993), 7-136, Internat. Press, Cambridge, MA, 1995.

[23] Hamilton, Richard S. A compactness property for solutions of the Ricci flow. Amer. J. Math. 117 (1995), no. 3, 545-572. 
[24] Hamilton, Richard S. Non-singular solutions of the Ricci flow on three-manifolds. Comm. Anal. Geom. 7 (1999), no. 4, 695-729.

[25] Hamilton, Richard; Isenberg, James. Quasi-convergence of Ricci flow for a class of metrics. Comm. Anal. Geom. 1 (1993), no. 3-4, 543-559.

[26] Heber, Jens. Noncompact homogeneous Einstein spaces. Invent. Math. 133 (1998), no. 2, $279-352$.

[27] Isenberg, James; Jackson, Martin. Ricci flow of locally homogeneous geometries on closed manifolds. J. Differential Geom. 35 (1992), no. 3, 723-741.

[28] Isenberg, James; Jackson, Martin; Lu, Peng. Ricci flow on locally homogeneous closed 4-manifolds. arXiv:math.DG/0502170.

[29] Ivey, Thomas. Ricci solitons on compact three-manifolds. Differential Geom. Appl. 3 (1993), no. 4, 301-307.

[30] Kleiner, Bruce; Lott, John. Notes on Perelman's papers. (25/05/2006 version) arXiv:math.DG/0605667.

[31] Knopf, Dan. Quasi-convergence of the Ricci flow. Comm. Anal. Geom. 8 (2000), no. 2, 375-391.

[32] Knopf, Dan; McLeod, Kevin. Quasi-convergence of model geometries under the Ricci flow. Comm. Anal. Geom. 9 (2001), no. 4, 879-919.

[33] Koiso, Norihito. On the second derivative of the total scalar curvature. Osaka J. Math. 16 (1979), no. 2, 413-421.

[34] Lauret, Jorge. Ricci soliton homogeneous nilmanifolds. Math. Ann. 319 (2001), no. 4, $715-733$

[35] Lauret, Jorge.; Will, Cynthia. Einstein solvmanifolds: existence and non-existence questions. arXiv:math.DG/0602502.

[36] Lunardi, A.; Metafune, G.; Pallara, D. Dirichlet boundary conditions for elliptic operators with unbounded drift. Proc. Amer. Math. Soc. 133 (2005), no. 9, 2625-2635.

[37] Lott, John. On the long-time behavior of type-III Ricci flow solutions. arXiv:math.DG/ 0509639.

[38] Milnor, John. Curvatures of left invariant metrics on Lie groups. Advances in Math. 21 (1976), no. 3, 293-329.

[39] Metafune, G.; Prüss, J.; Schnaubelt, R.; Rhandi. A. $L^{p}$-regularity for elliptic operators with unbounded coefficients. Adv. Differential Equations 10 (2005), no. 10, 1131-1164.

[40] Pazy, Ammon. Semigroups of linear operators and applications to partial differential equations. Applied Mathematical Sciences, 44. Springer-Verlag, New York, 1983.

[41] Perelman, Grisha. The entropy formula for the Ricci flow and its geometric applications. arXiv:math.DG/0211159.

[42] Perelman, Grisha. Ricci flow with surgery on three-manifolds. arXiv:math.DG/0303109.

[43] Petrunin, A.; Tuschmann, W. Diffeomorphism finiteness, positive pinching, and second homotopy. Geom. Funct. Anal. 9 (1999), no. 4, 736-774.

[44] Rabier, Patrick J. Elliptic problems on $\mathbb{R}^{N}$ with unbounded coefficients in classical Sobolev spaces. Math. Z. 249 (2005), no. 1, 1-30.

[45] Šešum, Nataša. Linear and dynamical stability of Ricci flat metrics. arXiv:math.DG 10410062.

[46] Shioya, Takashi; Yamaguchi, Takao. Collapsing three-manifolds under a lower curvature bound. J. Differential Geom. 56 (2000), no. 1, 1-66.

[47] Shioya, Takashi; Yamaguchi, Takao. Volume collapsed three-manifolds with a lower curvature bound. Math. Ann. 333 (2005), no. 1, 131-155.

[48] Schueth, Dorothee. On the 'standard' condition for noncompact homogeneous Einstein spaces. Geom. Dedicata 105 (2004), 77-83. 
(Christine Guenther) Pacific University

E-mail address: guenthec@pacificu.edu

(James Isenberg) University of Oregon

E-mail address: jim@newton.uoregon.edu

$U R L:$ http://physics.uoregon.edu/ $\mathrm{jim} /$

(Dan Knopf) University of Texas at Austin

E-mail address: danknopf@math.utexas.edu

URL: http://www.ma.utexas.edu/ danknopf/ 